\title{
Continuous Acoustic Monitoring of Electrical Machines; Processing Signals from USB Microphone \& Mobile Smartphone Sensors Detecting DC Motor Controller Fault
}

\author{
Jarek Grebenik*, Chris Bingham and Saket Srivastava \\ * The University of Lincoln, School of Engineering, Brayford Pool, Lincoln, LN6 7TS, UK \\ e-mail: \{jgrebenik, cbingham, ssrivastava\}@lincoln.ac.uk
}

\begin{abstract}
Transient current instability is one of the most common faults evident in Pulse Width Modulation (PWM) controlled brushless DC motors. This paper explores the underdeveloped field of real-time acoustic diagnostics for electrically based faults using consumer grade sensors. Current instabilities produce an audible torque transient on the motor, easily detectable using consumer acoustic sensors; a USB microphone and smartphone in this case. Two time-frequency signal processing techniques, Wavelet Packet Transform (WPT) and Empirical Mode Decomposition (EMD), are used to isolate information pertaining to the fault and are assessed for computational performance. This gives four processed signals to search for instabilities using a peak finding technique. We then compare the performance of each method. With the USB microphone WPT signal correlating the best results $(93 \%)$, a simplistic logarithmic predictive model is used to estimate the durations for the next experimental run, in real-time. The results prove that readily accessible and affordable consumer acoustic sensors can be used for real-time fault diagnostics with a high degree of accuracy.
\end{abstract}

Keywords: acoustic; electric; electrical; fault; detection; diagnosis; smartphone; consumer; microphone; motor; real-time; online; signal processing; wavelet packet transform; WPT; empirical mode decomposition; EMD; time-frequency analysis

Abbreviations: AE - Acoustic Emission, EEMD - Ensemble Empirical Mode Decomposition, EMD - Empirical Mode Decomposition, IMF - Intrinsic Mode Function, PWM - Pulse Width Modulation, WPT - Wavelet Packet Transform

\section{INTRODUCTION}

Acoustic supervision and monitoring is an attractive prospect for many industrial applications with proven advantages over many established systems including; earlier and more accurate detection, non-invasive and readily accessible sensors and better cost-benefit performance [1]. Research in this field is highly active with the majority focussed on seeded mechanical impact based faults, detecting the acoustic shockwave.

Acoustic Emission (AE) energy indexing/analysis has been used to detect seeded mechanical defects on roller element bearings $[2,3]$. Similar case study is also explored in $[4,5,6]$ using wavelet analysis. A good example of vibration analysis for bearing fault detection using wavelet transform is [7]. Another study investigates seeded mechanical defects on roller element bearings and gears using EMD of acoustic signals [8]. The work in [9] uses wavelet signal processing of vibration and acoustic signals to detect a simulated cracked tooth in a gear box. Another study mentions the requirement for real-time condition monitoring in industry and looks at motor fault diagnosis. However, it is unclear whether the experiment demonstrates real-time detection, and this is an area that is highly underdeveloped in this field [10]. All prior works discussed use specialised, research grade AE sensors, not easily accessible, that require mounting to the component to deliver sufficient signal to noise ratio. A review of condition monitoring and fault diagnostics for electrical motors is given in [11]. However, all the faults in this work are seeded and there is little mention of acoustic techniques. There is very little literature on acoustic diagnostics for electrically-based faults nor for real-time processing.

As opposed to seeded mechanical faults, it is far more difficult to detect and model transient current instabilities arising in PWM-based DC motors. Electronically commutated motors require a closed-loop power controller to convert the DC supply to AC for each phase; synchronising the motor. The design and operation of each controller will vary depending on the supply, type of motor and application. Often, controllers under commissioning, motor tuning, or operating in widely varying conditions, can result in transient current instabilities delivered to the motor. These instabilities could potentially cause damage to the controller or demagnetisation of the motor [12]. Detection could allow intervention to prevent further damage, and diagnostics could help assess maintenance requirements.

We present a novel application of acoustic monitoring to detect electro-mechanical instabilities on a brushless DC electric motor. Our work explores aspects previously unaddressed in the literature, namely; real-time processing and expansion to include electrically based faults. Consumer sensors (which are easily accessible) are used to highlight the under-usage of these types of sensor in both research and industry. Acoustic signals are processed in real-time to reveal the instability time-frequency information. This is done using two signal processing techniques, WPT and EMD, which are analysed for computational performance and ability to deliver information pertaining to the fault.

Our proposed solution uses a bespoke PWM power control system that delivers intermittent transient current instabilities with increasing frequency for higher voltages. At the end of the previous PWM cycle, the controller calculates the new duty from the demand. When the sampled current is already too high, the next cycle is switched off. This can resolve or continue to the end of the commutation period. A detailed view of the instability is given in Figure 1. This fault is native to this particular setup and is used here as an example of the potential electro-mechanical faults requiring diagnostic information.

Acoustics are well suited to this application due to the audible torque transient caused by the current instabilities; hence an electro-mechanical fault. In this case, the frequency 


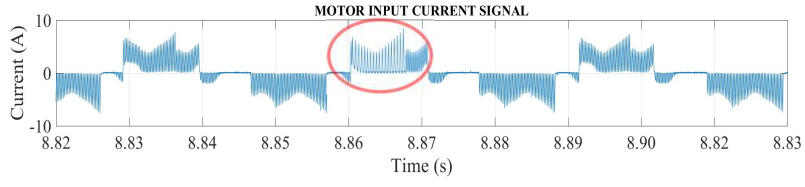

Figure 1: Motor current signal showing instability \#4 at the centre.

information is almost entirely in the audible range of a human ear. This permits the use of consumer sensors which are more accessible and can be remote from the workpiece. Should faults deliver ultrasonic frequencies then contact based sensors would likely be required.

In the next section, we describe the experimental setup and signal processing techniques employed. Detail is given on the design of WPT and EMD as used in the experiment, complete with an explanation on the real-time processing for this work. The experimental results are discussed with focus on; the diagnostic performance of the acoustic sensors and signal processing techniques, and real-time computational processing efficiency. The main findings, their impact and significance are conferred in the conclusions.

\section{METHODOLOGY}

This section describes and justifies the research approach; providing information regarding the experimental setup, data acquisition and signal processing. Two experimental runs are undertaken; the preliminary run gathers initial data allowing design of a predictive diagnostic model. This model is used to estimate instability duration throughout the primary run, in which processing is undertaken in real-time. The experimental setup for both runs is identical; the only minor improvements made to the preliminary run was the addition of real-time processing for predictive diagnostics.

\section{A. Experimental Setup \& Data Acquisition}

The experimental setup is given in Figure 2. A Unite MY1020 $1600 \mathrm{~W}, 36 \mathrm{~V}$ brushless DC motor is controlled using a custom Arduino-based digital PWM controller; consisting of two boards (low and high power). These are powered by a Keysight E3631A triple output DC PSU, set to deliver a voltage range of -15 to $+15 \mathrm{~V}$ and a Voltcraft PS1302D $0-30 \mathrm{~V}, 2 \mathrm{~A}$ PSU. The controller setup delivers a $10 \mathrm{kHz}$ current to drive the motor; speed is controlled by adjusting the supply voltage. High voltages or rapid voltage ramp up delivers intermittent transient instabilities. A USB microphone (Audio-Technica AT2020 USB+) and a smartphone (Samsung Galaxy S7 using WO Mic) are USB connected to a laptop; sampled by Matlab at $48 \mathrm{kHz}$ with 16 bit resolution. Both sensors are placed approximately $10 \mathrm{~cm}$ away with their sensitive planes aimed toward the motor. A single phase of the motor input current from a Halleffect sensor control feedback loop is sampled at $250 \mathrm{kHz}$ with 16 bit resolution using a Tektronix DPO 7054C oscilloscope.

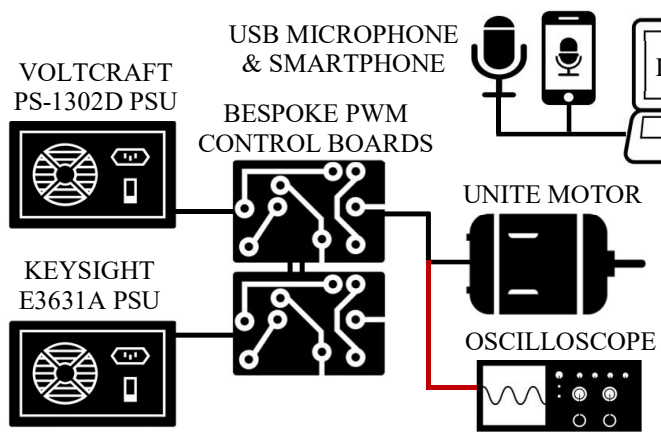

Figure 2: Diagram of the experimental setup.

The practical operation of the sampling is complex; both the audio and current sampling must be triggered manually. The smartphone audio signal lags the USB microphone signal by approximately $0.5 \mathrm{~s}$ due to the smartphone audio streaming software. To capture sufficient instabilities a sampling duration of $20 \mathrm{~s}$ is chosen based on previous experience. The oscilloscope is set to sample for $20 \mathrm{~s}$. To accommodate for the time delay from manual triggering and the smartphone lag, the audio sampling duration is set to $25 \mathrm{~s}$. The audio is triggered first, followed by the oscilloscope within the $5 \mathrm{~s}$ window. This allows for the audio and current signals to be synchronised in post processing. The Voltcraft control voltage for each run was slightly different (approximately $21 \mathrm{~V}$ for the first run and $23 \mathrm{~V}$ for the second run), as creating the conditions for instabilities to manifest is difficult to control.

\section{B. Signal Processing \& Analysis}

For the preliminary run, all data streams are synchronised manually in the time-domain. This is repeated for analysis purposes in post for the primary run. Figure 3 shows the raw unprocessed signals from the oscilloscope current, and microphone and smartphone audio.

The aim of signal processing is to extract and separate the fault information from the background noise as much as possible. The instability can be detected by finding a spike in the audio signal indicating the louder ticking sound of the torque transient. However, the time domain information is noisy, meaning a peak finding technique could either miss instabilities due to insufficient amplitude, or have erroneous detection due to background noise. By analysing the timefrequency domain, much of the background noise can be removed with complex filtering. A filtered signal can then be analysed for spikes indicative of instabilities.

The preliminary run audio signals are used to design the WPT and EMD signal processing parameters, the peak finding detection method and some simplistic diagnostics. This design is performed through post-analysis, not in realtime. The designed method is then implemented for the main run in real-time. WPT and EMD $[5,13]$ are selected for testing 

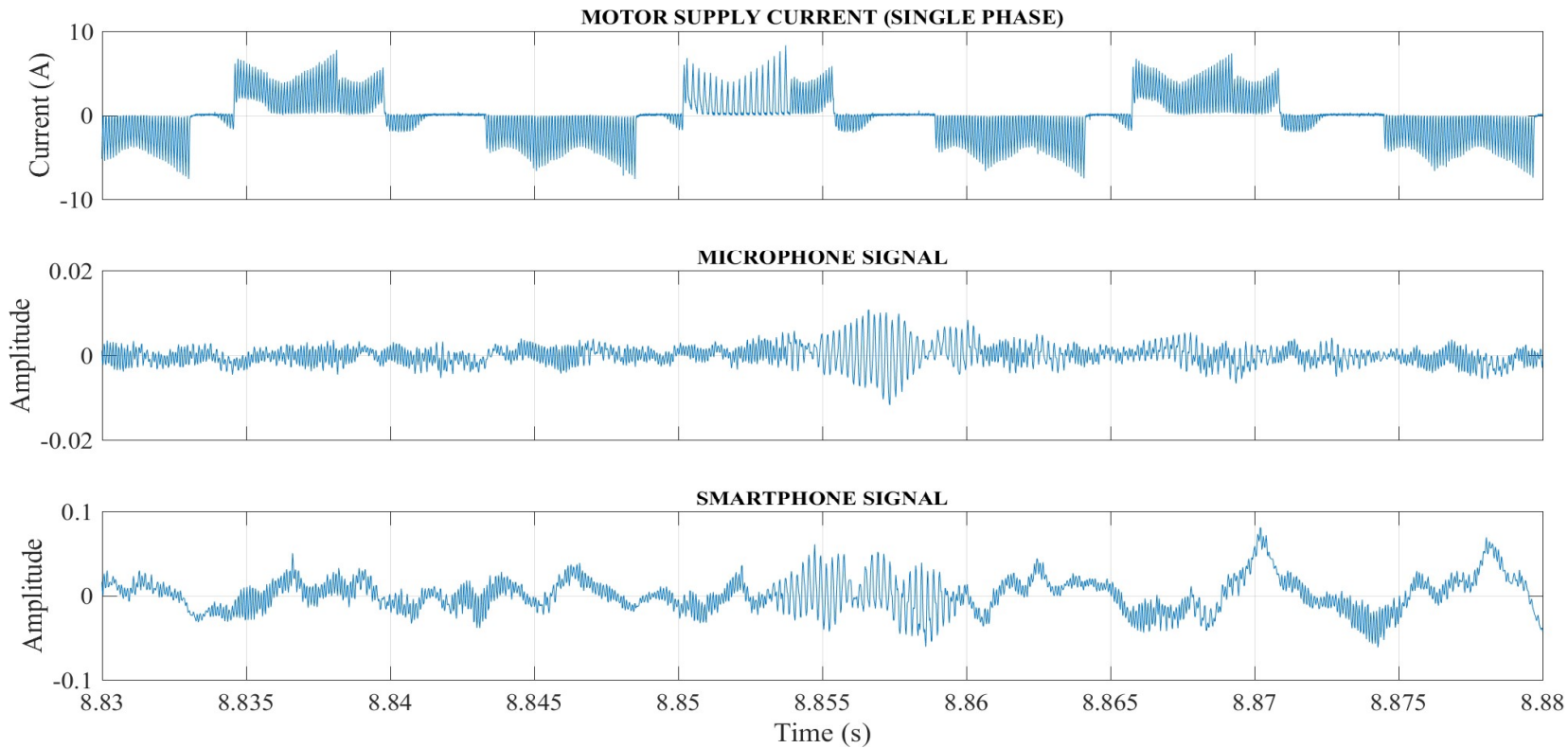

Figure 3: Plot of the synchronised raw signals showing instability \#6. Note the units of the audio signal are vacillating as only the relative changes in amplitude that are of interest.

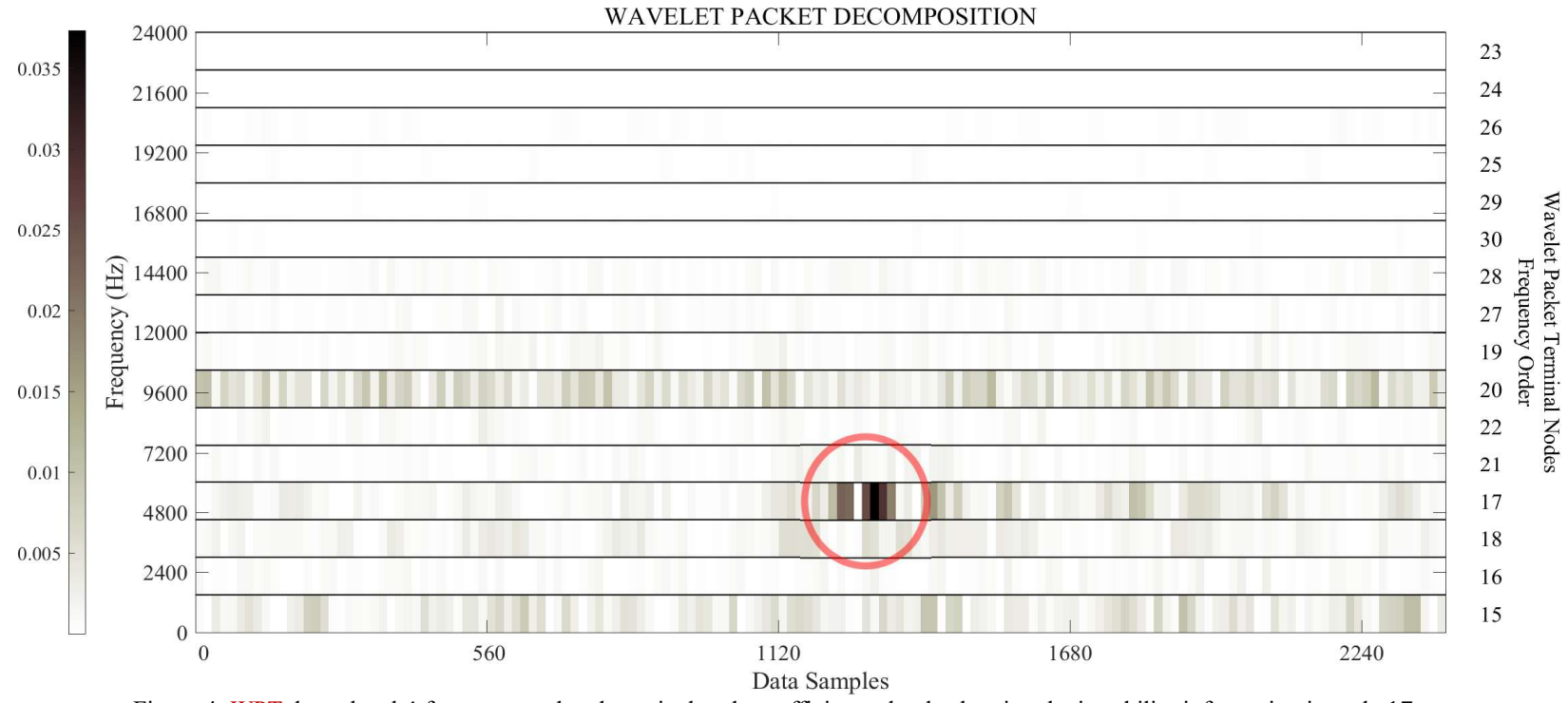

Figure 4: WPT dmey level 4 frequency ordered terminal node coefficients clearly showing the instability information in node 17.

as they are prominent time-frequency techniques in acoustic diagnostic literature. WPT allows for complex filtering to accurately reconstruct the fault information whilst leaving out unwanted noise $[4,5,6]$. EMD decomposes a signal by sifting the mean of the upper and lower envelopes into IMFs. This technique is more akin to an empirical algorithm rather than a theoretical transform; however, this makes it especially good with non-stationary and non-linear signals; an ideal candidate for fault detection [8, 13]. EEMD [14] is not required as audio is highly oscillatory by nature. Furthermore, the additional processing is highly computationally intensive and unsuitable for real-time processing.

\section{1) Wavelet Packet Transform Design}

The parameters that require selection and optimisation are; the selection of the mother wavelet, the number of levels of decomposition and, selection of the reconstruction node. The mother wavelet is chosen based on the maximum signal energy to Shannon-entropy ratio as detailed in [15]. 60 different mother wavelets from the Haar, Daubechies, Symlets, Coiflets, Biorthogonal, Reverse-Biorthogonal, Discrete approximation of Meyer (dmey) and Fejer-Korovkin families are analysed. The dmey wavelet gave very high performance; isolating nearly all the fault information into a single terminal node. The wavelet analyser is used to determine the required level of decomposition using the terminal node coefficients (Figure 4); aiming to maximise 

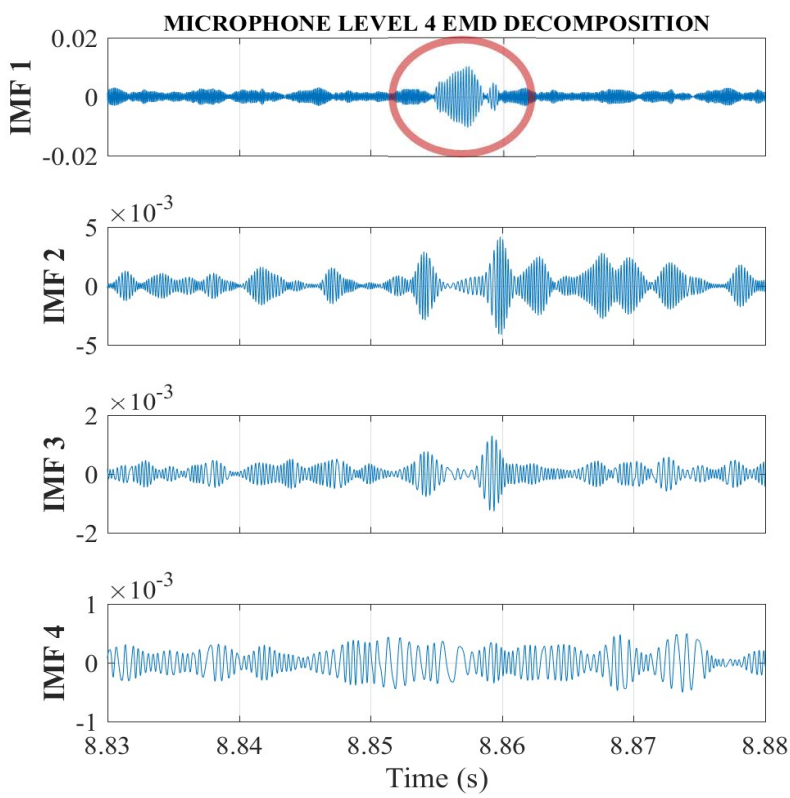
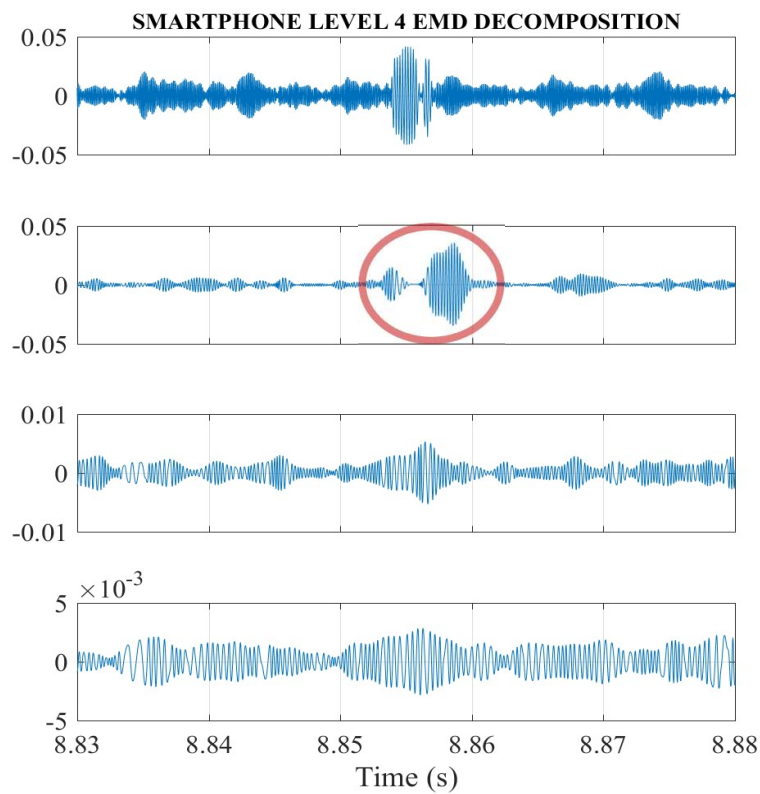

Figure 5: EMD level 4 decomposition of both the microphone (left) and smartphone (right) test signals.
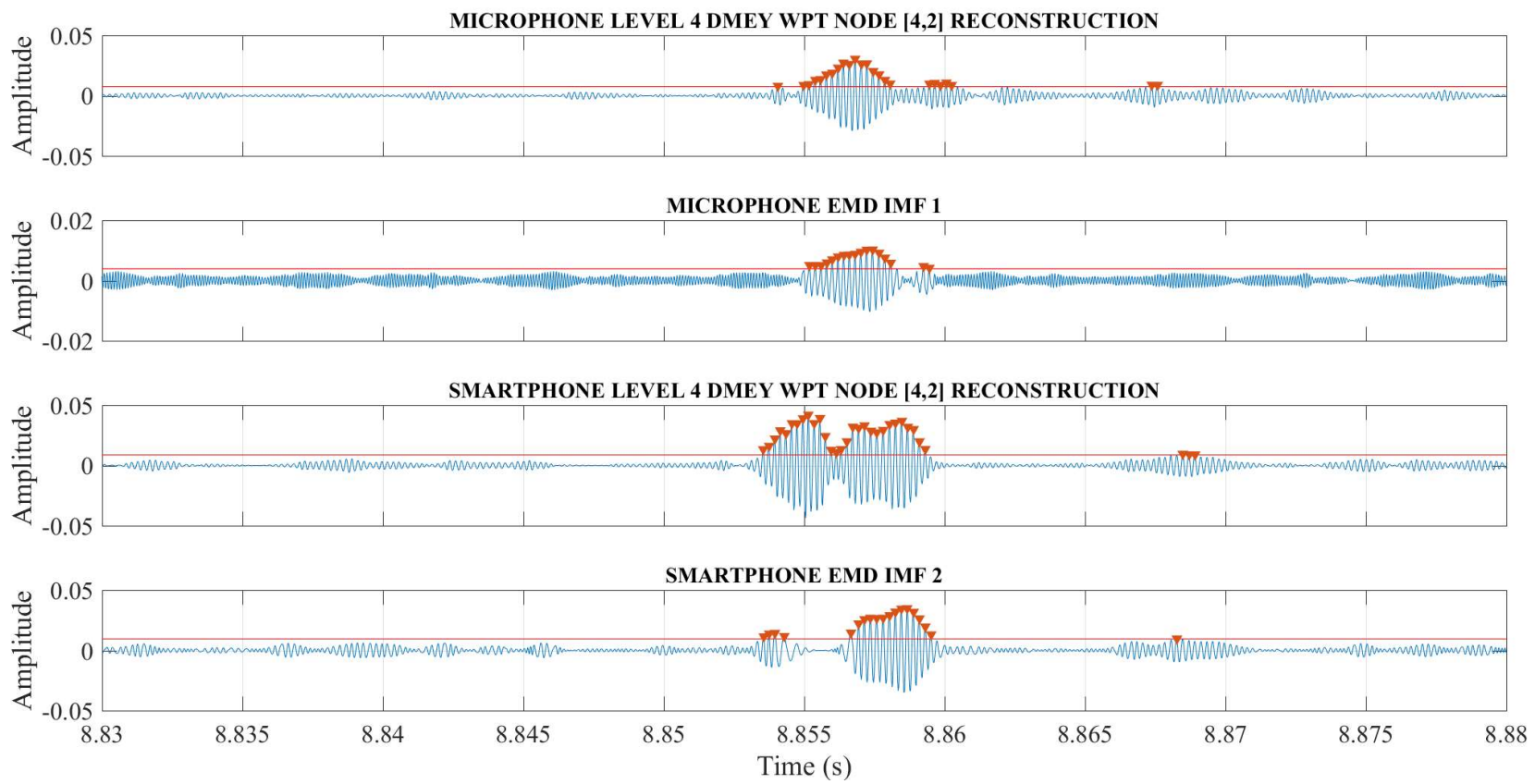

Figure 6: Microphone and smartphone signals processed by WPT and EMD. Threshold and the representative peaks are highlighted.

isolation of the fault information into the minimum number of nodes. This is clearly visible in node 17 as shown. The node's coefficients are used to reconstruct the signal; preserving the fault information and filtering out most of the unwanted noise.

\section{2) Empirical Mode Decomposition Design}

To determine the required level of decomposition the audio signals are initially decomposed to give $4 \mathrm{IMFs}$, shown in Figure 5. For the microphone and smartphone, IMF 1 and IMF 2 are selected respectively for further processing. This is based on visual analysis showing the best separation of the fault information away from background noise.

\section{3) Instability Detection Design}

To summarise, the original microphone and smartphone signals (Figure 3 ), are processed through WPT and EMD to give $2 \times 2$ signals (shown in Figure 6):

- microphone WPT

- smartphone WPT

- microphone EMD IMF 1

- smartphone EMD IMF 2.

The fault information signal to noise ratio is significantly improved. A standard peak finding algorithm detects the instability spikes in each processed signal. A standard deviation based amplitude threshold, is set for each signal such that only instability spikes protrude above. The key to diagnostic tuning is setting the threshold such that sufficient 
width is detected to estimate the duration. Windowing discretises between separate instabilities (requiring a minimum separation of $0.02 \mathrm{~s}$ ). This value is chosen based on observation to be sufficient to distinguish between different instabilities but, not so long as to class two separate instabilities together. Figure 6 shows the threshold line and separate instabilities highlighted.

\section{4) Diagnostics Design}

Basic diagnostics are performed to illustrate the potential for acoustics for similar applications. For each processed signal (Figure 6), the duration between the first and last peak found for a single instability is correlated against the actual duration (derived from the current signal) according to Eq. 1.

$$
\rho_{X, Y}=\frac{\sum(x-\bar{x})(x-\bar{y})}{\sqrt{\sum(x-\bar{x})^{2} \sum(x-\bar{y})^{2}}}
$$

A simplistic logarithmic model is developed, based on the best performing correlation results (in this case microphone WPT), to predict the instability duration in real-time throughout the primary run.

\section{5) Real-Time Processing}

To bound the problem, the purpose of real-time processing is to detect faults quickly enough to either shutdown the motor or provide a warning to the operator. Real-time processing can either be achieved using analog or digital techniques. Analog processing by nature performs much faster than digital, but this is beyond the scope of this work. Digital processing relies on splitting the data into discretised time segments. The previous segment can be searched for faults whilst the next is recorded. For continuous operation, this relies on the processing time being shorter than the time duration of each segment. The segment duration determines the refresh rate and detection response time of the system.

In this case, the refresh rate is set to $0.2 \mathrm{~s}$, fast enough for all intended purposes and appearing real-time to the user. As the next segment is recorded the previous one is searched for instabilities as per the methods described above. The primary run current signal and audio are synchronised and processed identically to the preliminary run. The diagnostic results are analysed for accuracy against the actual instabilities and their durations. The results are presented in the next section.

\section{RESULTS \& DISCUSSION}

This work set out to push the boundary of acoustic monitoring into diagnosing electrically-based faults; this case uses a transient current instability on an electric motor by way of example. By using consumer sensors, the practical uses these devices can deliver are highlighted. The diagnostic and computational performance of real-time WPT and EMD signal processing is examined.

\section{A. Acoustics \& Sensors Diagnostic Performance}

The preliminary run first to last peak durations are given in Table I. For the 10 instabilities that occurred, all were detected by each processed signal. Table II gives the same results from the primary run where 82 instabilities occurred; some have been omitted for brevity. The greater number of
TABLE I: THE FIRST TO LAST PEAK DURATION OF EACH PEAK CLUSTER FROM THE PROCESSED AUDIO SIGNALS CORRELATED AGAINST THE ACTUAL INSTABILITY DURATION DERIVED FROM THE CURRENT SIGNAL ANALYSIS.

\section{RUN 1 FIRST TO LAST PEAK DURATIONS (SECONDS)}

\begin{tabular}{|c|c|c|c|c|c|}
\hline \multirow{2}{*}{$\begin{array}{c}\text { Instability } \\
\text { Number }\end{array}$} & \multirow{2}{*}{ Current } & \multicolumn{2}{|c|}{ Microphone } & \multicolumn{2}{c|}{ Smartphone } \\
\cline { 3 - 6 } & & $\boldsymbol{W P T}$ & $\boldsymbol{E M D}$ & $\boldsymbol{W P T}$ & $\boldsymbol{E M D}$ \\
\hline 1 & 0.003 & 0.014 & 0.003 & 0.024 & 0.019 \\
\hline 2 & 0.003 & 0.014 & 0.003 & 0.019 & 0.015 \\
\hline 3 & 0.003 & 0.007 & 0.003 & 0.009 & 0.009 \\
\hline 4 & 0.002 & 0.002 & 0.000 & 0.008 & 0.007 \\
\hline 5 & 0.003 & 0.013 & 0.003 & 0.017 & 0.018 \\
\hline 6 & 0.003 & 0.014 & 0.004 & 0.015 & 0.015 \\
\hline 7 & 0.003 & 0.014 & 0.003 & 0.028 & 0.019 \\
\hline 8 & 0.003 & 0.014 & 0.003 & 0.019 & 0.019 \\
\hline 9 & 0.001 & 0.002 & 0.001 & 0.008 & 0.007 \\
\hline 10 & 0.001 & 0.001 & 0.001 & 0.006 & 0.009 \\
\hline \multicolumn{2}{|c|}{ Correlation $\mathbf{( \% )}$} & $\mathbf{9 3 . 3 8}$ & $\mathbf{7 5 . 3 1}$ & $\mathbf{8 1 . 7 0}$ & $\mathbf{8 2 . 2 5}$ \\
\hline
\end{tabular}

TABLE II: SUMMATIVE TABLE OF THE FIRST TO LAST PEAK DURATION FROM THE SECOND REAL-TIME RUN.

\begin{tabular}{|c|c|c|c|c|c|c|}
\hline \multicolumn{6}{|c|}{$\begin{array}{l}\text { RUN } 2 \text { FIRST TO LAST PEAK DURATIONS } \\
\text { (SECONDS) }\end{array}$} & \multirow{3}{*}{$\begin{array}{c}\text { LOGARITHMIC } \\
\text { ESTIMATION } \\
\text { ERROR (\%) }\end{array}$} \\
\hline \multirow{2}{*}{$\begin{array}{l}\text { Instability } \\
\text { Number }\end{array}$} & \multirow{2}{*}{ Current } & \multicolumn{2}{|c|}{ Microphone } & \multicolumn{2}{|c|}{ Smartphone } & \\
\hline & & $W P T$ & $E M D$ & $W P T$ & $E M D$ & \\
\hline 1 & 0.003 & 0.005 & 0.004 & 0.014 & 0.013 & -12.957 \\
\hline 2 & 0.003 & 0.005 & 0.004 & 0.013 & 0.014 & -11.814 \\
\hline 3 & 0.003 & 0.004 & 0.004 & 0.013 & 0.009 & -22.268 \\
\hline 4 & 0.003 & 0.005 & 0.004 & 0.018 & 0.013 & -12.957 \\
\hline 5 & 0.003 & 0.005 & 0.004 & 0.013 & 0.011 & -14.548 \\
\hline 11 & 0.003 & 0.005 & 0.003 & 0.009 & 0.009 & -14.412 \\
\hline 44 & 0.001 & 0.002 & - & 0.009 & 0.009 & 48.877 \\
\hline 60 & 0.001 & 0.008 & 0.008 & 0.024 & 0.007 & 376.768 \\
\hline 81 & 0.003 & 0.005 & 0.004 & 0.009 & 0.009 & -12.957 \\
\hline 82 & 0.003 & 0.005 & 0.004 & 0.014 & 0.009 & -14.548 \\
\hline Min & 0.001 & 0.002 & 0.003 & 0.006 & 0.001 & -25.692 \\
\hline Max & 0.003 & 0.008 & 0.008 & 0.025 & 0.021 & 376.768 \\
\hline Mean & 0.003 & 0.005 & 0.004 & 0.012 & 0.010 & -1.209 \\
\hline Std. Dev. & 0.001 & 0.001 & 0.001 & 0.004 & 0.003 & 64.941 \\
\hline
\end{tabular}

instabilities is due to the higher control voltage. All instabilities were detected by each processed signal; with a single exception of microphone EMD instability 44, one of the shortest that occurred. There were no erroneous detections in either run. This issue has been partially addressed through complex time-frequency filtering, but minimising false alarms is beyond the scope of this work.

With almost perfect detection rates for all processed signals, the consumer sensors and time-frequency signal processing techniques are ideally suited for remote monitoring of electrical machines. To expand on this achievement, additional information, providing a diagnostic element, can be extracted from the acoustic signals. Estimating the instability duration from the acoustic signal can indicate whether preventative or corrective intervention is required. By way of example, Table II also gives the logarithmic model (based on the microphone WPT signal) estimation error. Demonstrating that, despite its simplicity; based on a small preliminary data set with no validation, still accurately predicted the durations with only $-1.2 \%$ mean error. However, due to the limitations of the model and training data the spread is quite high. Nevertheless, it still illustrates that acoustics has huge potential for diagnostic capabilities. 
TABLE III: FIRST TO LAST PEAK DURATION CORRELATIONS.

\begin{tabular}{|c|c|c|c|}
\hline$(\%)$ & WPT & EMD & Average \\
\hline Microphone & 93.38 & 75.31 & 84.35 \\
\hline Smartphone & 81.70 & 82.25 & 81.97 \\
\hline Average & 87.54 & 78.78 & 83.16 \\
\hline
\end{tabular}

TABLE IV: AVERAGE FRAME PROCESSING TIMES ACROSS ALL FRAMES AND FOR EACH SIGNAL PROCESSING METHOD.

\begin{tabular}{|c|c|c|c|c|}
\hline \multirow{2}{*}{} & \multicolumn{4}{|c|}{ FRAME PROCESSING TIME (SECONDS) } \\
\cline { 2 - 5 } & $\boldsymbol{M E A \boldsymbol { N }}$ & $\boldsymbol{S T D} \boldsymbol{D E} \boldsymbol{V}$ & $\boldsymbol{M A} \boldsymbol{X}$ & $\boldsymbol{M I N}$ \\
\hline WPT & 0.2034 & 0.0454 & 0.6205 & 0.1460 \\
\hline EMD & 0.2037 & 0.0495 & 0.6554 & 0.1189 \\
\hline
\end{tabular}

Table III gives the correlation results from the preliminary data showing that the microphone sensor and WPT processing performed the best with $84.4 \%$ and $87.5 \%$ correlations respectively. The best overall correlation of $93.4 \%$, naturally comes from the microphone signal processed using WPT.

The smartphone performance compared to the microphone is particularly impressive considering the aperture and diaphragm are considerably smaller. The technology currently present in smartphones prevents them from becoming an allin-one diagnostic tool, although this is likely to change as processing and memory improves. Comparing to analysing the current signal, these methods provide a simple and costeffective solution. Although current analysis would provide better accuracy, due to the high sampling rates and specialist knowledge required it is significantly more difficult.

\section{B. Real-Time Acoustic Fault Diagnosis - EMD \& WPT Performance Comparison}

WPT outperformed EMD both diagnostically and computationally. WPT isolated almost all the fault information within a single terminal node, delivering a wellfiltered signal and improved performance. EMD split some information between the IMFs due to the nature of sifting. The computation required for EMD is greater than WPT, despite the inclusion of the duration prediction. Table IV gives the processing times that indicate which is more computationally intensive. Despite a minute time difference of $-3.42 \times$ $10^{-4} \mathrm{~s}$, this represents a significant cost saving over long periods. Note that the processing time for each frame is slightly longer than the recording time meaning this system will only run up to the limits of the computer memory. For a continuous system, faster processing would be required so the frame processing time is smaller than the recording time.

\section{CONCLUSION}

Real-time acoustic fault diagnosis, of an electrically-based fault, using consumer sensors, is demonstrated on a transient current instability for a PWM controlled brushless DC motor. $100 \%$ of the instabilities were detected with the microphone sensor and WPT processing proving the strongest candidates. This broadens the horizon for acoustic monitoring, previously limited to seeded mechanical faults, to include electricallybased faults. Acoustic processing in real-time, seriously under-developed in the literature, is performed digitally. This aspect is crucial for industry adoption of acoustic measurements for monitoring and supervision purposes. Here, accessible laptop hardware is used, easily achieving real-time processing. The consumer sensors show that research grade costly sensors are not required until fault information is contained in ultrasonic frequencies. These sensors have added advantages; being remote from the workpiece, able to monitor components with limited or no access, excellent accuracy and diagnostic capabilities as well cost-benefit performance. The historic knowledge, confidence and reliance surrounding established condition monitoring techniques such as vibration has resulted in the uptake of acoustics by industry to be very slow. The methods demonstrated are applicable to almost any fault with an acoustic signature. It is hoped that this work could open opportunities for future development of this field.

\section{REFERENCES}

[1] D. Mba and R. Rao, "Development of acoustic emission technology for condition monitoring and diagnosis of rotating machines; bearings, pumps, gearboxes, engines and rotating structures," The Shock and Vibration Digest, vol. 38, no. 1, pp. 3 - 16, 2006.

[2] K. Al-Balushi, A. Addali, B. Charnley and D. Mba, "Energy Index technique for detection of Acoustic Emissions associated with incipient bearing failures," Applied Acoustics, vol. 71, no. 9, pp. 812 - 821, 2010.

[3] S. Al-Dossarya, R. Raja Hamzahb and D. Mba, "Observations of changes in acoustic emission waveform for varying seeded defect sizes in a rolling element bearing," Applied Acoustics, vol. 70, no. 1, pp. 58 - 81, 2009.

[4] P. Konar and P. Chattopadhyay, "Bearing fault detection of induction motor using wavelet and support vector machines (SVMs)," Applied Soft Computing, vol. 11, no. 6, pp. 4203 - 4211, 2011.

[5] N. Nikolaou and I. Antoniadis, "Rolling element bearing fault diagnosis using wavelet packets," NDT\&E International, vol. 35, no. 3, pp. 197 $205,2002$.

[6] F. Hemmati, W. Orfali and M. Gadala, "Roller bearing acoustic signature extraction by wavelet packet transform, applications in fault detection and size estimation," Applied Acoustics, vol. 104, pp. 101 - 118, 2016.

[7] P. Kankar, S. C. Sharma and S. Harsha, "Rolling element bearing fault diagnosis using wavelet transform," Neurocomputing, vol. 74, no. 10, pp. $1638-1645,2011$.

[8] M. Amarnath and I. R. P. Krishna, "Empirical mode decomposition of acoustic signals for diagnosis of faults in gears and rolling element bearings," IET Science, Measurement and Technology, vol. 6, no. 4, pp. 279 - 287, 2011.

[9] T. Loutas, G. Sotiriades, I. Kalaitzoglou and V. Kostopoulos, "Condition monitoring of a single-stage gearbox with artificially induced gear cracks utilizing on-line vibration and acoustic emission measurements," Applied Acoustics, vol. 70, no. 9, pp. 1148 - 1159, 2009.

[10] M. Elmaleeh, N. Saad and M. Awan, "Condition monitoring of industrial process plant using acoustic emission techniques," in 2010 International Conference on Intelligent and Advanced Systems (ICIAS), Kuala Lumpur, 2010.

[11] S. Nandi, H. A. Toliyat and X. Li, "Condition Monitoring and Fault Diagnosis of Electrical Motors - A Review," IEEE Transactions On Energy Conversion, vol. 20, no. 4, pp. 719 - 729, 2005.

[12] M. Riera-Guasp, J. A. Antonino-Daviu and G.-A. Capolino, “Advances in electrical machine, power electronic, and drive condition monitoring and fault detection: state of the art," IEEE Transactions On Industrial Electronics, vol. 62, no. 3, pp. 1746 - 1759, 2015.

[13] N. Huang, Z. Shen, S. Long, M. Wu, H. Shih, Q. Zheng, N.-C. Yen, C. C. Tung and H. Liu, "The empirical mode decomposition and the Hilbert spectrum for nonlinear and non-stationary time series analysis," The Royal Society Proceedings A - Mathematical, Physical and Engineering Sciences, vol. 454, pp. 903 - 995, 1998.

[14] Z. Wu and N. Huang, "Ensemble Empirical Mode Decomposition - A Noise Assisted Data Analysis Method," Advances in Adaptive Data Analysis, vol. 1, no. 1, pp. 1 - 41, 2009.

[15] R. Yan, "Wavelet selection criteria for non-stationary vibration analysis in bearing health diagnosis," University of Massachusetts, 2007. 